\title{
Magnetic sentinel node and occult lesion localization in breast cancer (MagSNOLL Trial)
}

\author{
M. Ahmed ${ }^{1,2}$, B. Anninga ${ }^{1}$, S. Goyal ${ }^{4}$, P. Young ${ }^{5}$, Q. A. Pankhurst ${ }^{3}$ and M. Douek ${ }^{1,2}$, on behalf of the \\ MagSNOLL Trialists Group* \\ ${ }^{1}$ Research Oncology, Division of Cancer Studies, King's College London, ${ }^{2}$ Department of Breast Surgery, Guy's and St Thomas' NHS Foundation Trust, \\ and ${ }^{3}$ Institute of Biomedical Engineering, University College London, London, and Departments of ${ }^{4}$ Breast Surgery and ${ }^{5}$ Breast Radiology, University \\ Hospital Llandough, Cardiff, UK \\ Correspondence to: Mr M. Douek, Research Oncology, Division of Cancer Studies, King's College London, 3rd Floor Bermondsey Wing, Guy's Hospital, \\ Great Maze Pond, London SE1 9RT, UK (e-mail: michael.douek@kcl.ac.uk)
}

\begin{abstract}
Background: Non-palpable breast cancers require localization-guided surgery and axillary staging using sentinel lymph node biopsy (SLNB). This study investigated the novel technique of magnetic-guided lesion localization and concurrent SLNB, which avoids the need for wire-guided localization and radioisotopes.

Methods: An ultrasound-guided intratumoral injection of magnetic tracer $(0.5 \mathrm{ml})$ was performed in a protocol-driven predefined minimum of ten patients with palpable breast cancer to assess the ability of the magnetic tracer safely to localize the tumour at the site of injection and concurrently drain to the lymphatics. Once successful lesion localization had been confirmed (peak magnetometer count retained at the centre of the tumour), the technique was undertaken in a further 20 patients with non-palpable breast cancers awaiting wide local excision and SLNB. All patients underwent SLNB with both the magnetic and standard dual (radioisotope and Patent Blue V dye) techniques.

Results: Thirty-two patients were recruited, of whom 12 (1 with bilateral disease) presented with palpable and 20 with non-palpable breast cancer. Peak magnetometer counts were retained at the tumour centre in all palpable (13) and non-palpable (20) breast cancers. Re-excisions for involved margins were necessary in two patients with non-palpable breast cancers. The sentinel lymph node identification rates were 28 of 33 procedures for the magnetic technique alone, 32 of 33 for the magnetic technique combined with blue dye, and 32 of 33 for the standard dual technique.

Conclusion: Magnetic lesion localization is feasible, with intratumoral magnetic tracer injection combined with a periareolar injection of blue dye for subsequent SNLB.

* Other members of the MagSNOLL Trialists Group are co-authors of this study and can be found under the heading Collaborators

Presented to the 37th Annual Breast Cancer Symposium, San Antonio, Texas, USA, December 2014

Paper accepted 4 February 2015

Published online in Wiley Online Library (www.bjs.co.uk). DOI: 10.1002/bjs.9800
\end{abstract}

\section{Introduction}

One-third of all breast cancers are non-palpable at the time of diagnosis ${ }^{1}$. The current standard treatment for these clinically occult lesions is surgical excision by wire-guided localization and axillary staging using sentinel lymph node biopsy (SLNB). Despite being the standard of care, wire-guided localization has significant drawbacks, including technical difficulties, poor cosmetic outcome, diathermy burns and high reoperation rates. Consequently, alternative techniques have been developed including radioguided localization and intraoperative ultrasonography. These techniques have demonstrated benefits over wire-guided localization in systematic reviews and meta-analyses ${ }^{2,3}$. However, their uptake has been limited owing to dependence on radioisotope with its associated accessibility, legislative and handling issues, and the difficulties in formal ultrasound training for surgeons ${ }^{4}$. The technique of SLNB has also consequently been vastly underused because of its dependence on radioisotopes, with less than 60 per cent of eligible patients in the developed world having access to the procedure ${ }^{5}$.

A novel magnetic technique was developed for $\mathrm{SLNB}^{6}$ and this formed the basis for the SentiMAG Multicentre 


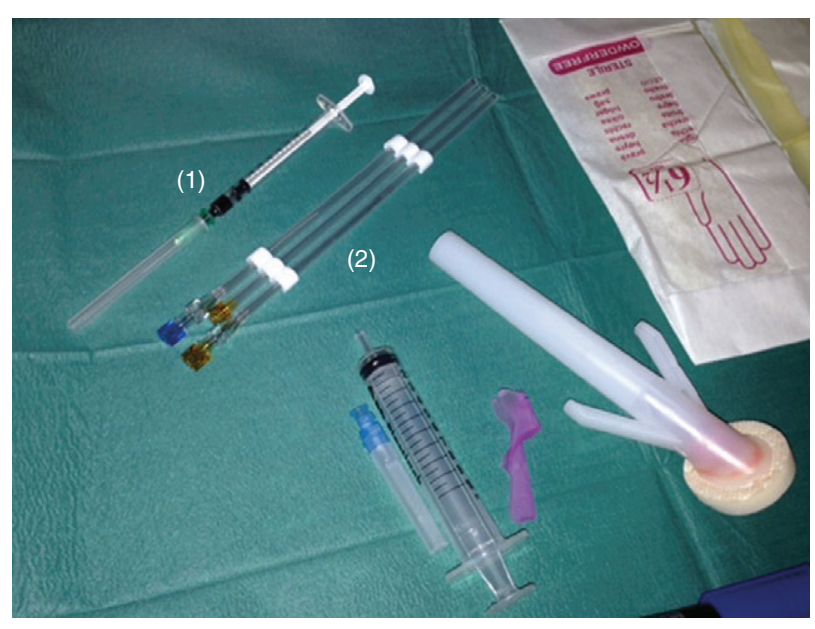

a Equipment

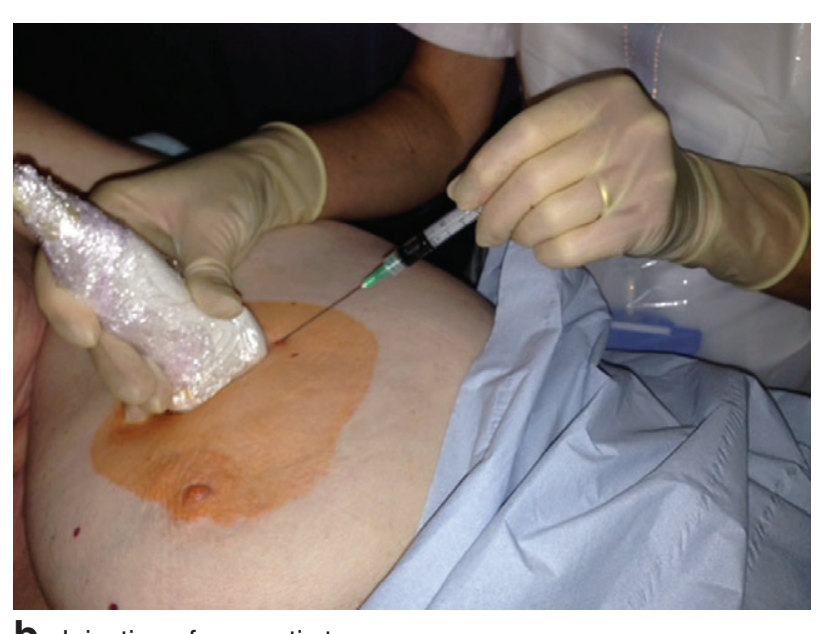

b Injection of magnetic tracer

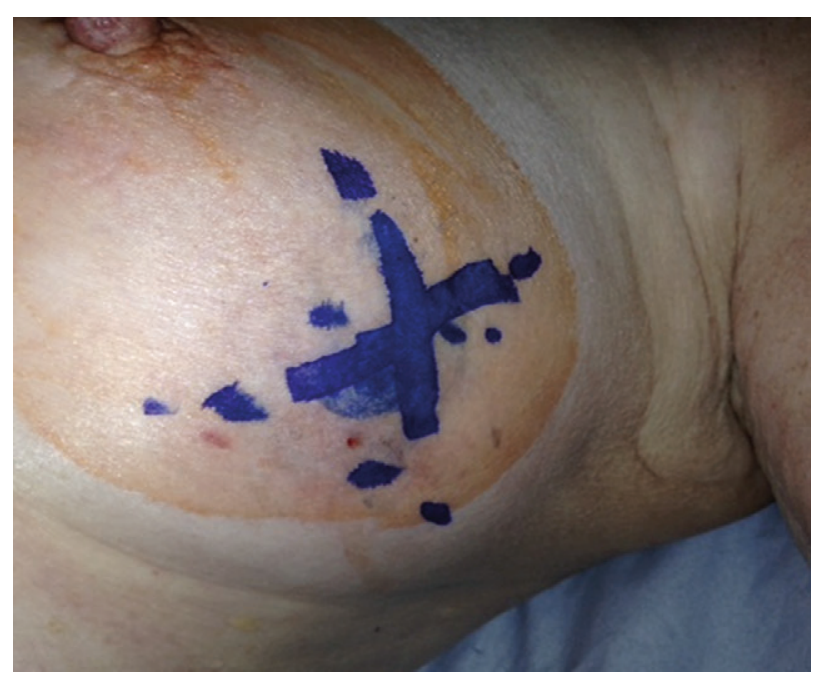

Skin marking over lesion
Trial $^{7}$, which demonstrated the non-inferiority of the magnetic technique for SLNB detection in breast cancer compared with the standard 'dual technique' comprising use of radioisotope and blue dye. The findings of the SentiMAG Multicentre Trial $^{7}$ were subsequently confirmed by the Central-European SentiMAG Study ${ }^{8}$. Superparamagnetic iron oxide (SPIO) has been used to perform SLNB in breast cancer ${ }^{7-9}$, and is one of the most promising radioisotope-free techniques ${ }^{10}$. Once injected interstitially into the breast, the SPIO travels to the axillary lymph nodes and is distributed within the sinuses, subcapsular space and parenchyma of the nodes ${ }^{11}$. Iron is sequestered within macrophages before being broken down and distributed across iron stores in the body ${ }^{12}$. The magnetic tracer can be detected during surgery using a handheld magnetometer, allowing the SLNB independent of radioisotopes ${ }^{7-9}$.

The localization properties of the magnetic tracer were established in preclinical phantoms ${ }^{12}$, and an in vivo porcine model was developed and used successfully to validate the performance of magnetic SLNB and assessment of localization properties ${ }^{13}$. The Magnetic Sentinel Node and Occult Lesion Localization (MagSNOLL) Trial $^{14}$ evaluated the feasibility of a magnetic dye-directed lesion localization technique with concurrent SLNB, using the magnetic technique. The protocol-driven analysis of the first 20 patients with non-palpable breast cancers enrolled is presented here.

\section{Methods}

The study was conducted at units experienced with the use of the magnetic technique for SLNB in breast cancer. The magnetic radiological localization procedure and the surgical localization procedure with magnetic SLNB were standard for all participating radiologists and surgeons. Data collection was undertaken prospectively using HP Teleform (Hewlett-Packard Autonomy, Cambridge, UK) with either electronic clinical record forms or eForms. Ethics committee approval was granted for this study within the UK (13/LO/0636). The trial was registered

Fig. 1 Magnetic sentinel node and occult lesion localization procedure. a Injection of $0.5 \mathrm{ml}$ of magnetic tracer (1) is followed by placement of a non-ferromagnetic marker coil (2), solely for the purpose of facilitating specimen intraoperative radiography - not as part of the localization procedure. b Intratumoral injection $(0.5 \mathrm{ml}$ magnetic tracer $)$ administered under ultrasound guidance in the radiology suite. c Ultrasound-guided skin marking over the site of the breast cancer 
with the UK Clinical Research Network (ISRCTN 68689512, UKCRN 14979).

\section{Patient recruitment}

Between 4 August 2013 and 8 June 2014, patients with histologically confirmed invasive breast cancer visible on ultrasound imaging and suitable for SLNB (normal or indeterminate/abnormal preoperative axillary ultrasonography and benign fine-needle aspiration or core biopsy) were recruited, of whom a predefined minimum of ten of the first recruited patients had to have a palpable breast cancer. All patients had to be available for a minimum of 12 months' follow-up. Patients with intolerance or hypersensitivity to iron or dextran compounds, who could not or did not receive radioisotope for SLNB, suffered from iron overload disease, and had pacemakers or other implantable devices in the chest wall were excluded. In practice, none of the patients met any of the exclusion criteria. Potential patients were identified by investigators from multidisciplinary team meetings. An invitation letter was sent to eligible patients together with a patient information sheet. Patients who were willing to participate in the study provided informed written consent on the day of elective surgery or at a previous hospital visit. Details of all patients approached about the trial were recorded on the patient-screening log and kept in the investigator site files.

\section{Magnetic sentinel node and occult lesion localization}

Within $24 \mathrm{~h}$ of surgery the patients received an intratumoral injection of $0.5 \mathrm{ml}$ magnetic tracer (Sienna+ ${ }^{\circledR}$ (27 mg iron per ml); Endomagnetics, Cambridge, UK) and placement of a non-ferromagnetic marker coil under ultrasound guidance, followed by skin marking directly overlying the lesion (Fig. 1). Patients received the standard protocol of the combined technique (radioisotope and Patent Blue dye) for SLNB. SLNB was commenced, guided by a handheld magnetometer (SentiMAG ${ }^{\circledR}$; Endomagnetics); the $\gamma$ probe was used only after magnetometer-detected nodes had been identified and removed for ex vivo counts. The handheld magnetometer was used to localize the centre of the tumour and confirm that the peak magnetometer count corresponded to the skin marking placed by the radiologist. Surgical excision of the breast lesion was undertaken. Once the surgeon was satisfied that the lesion had been excised with satisfactory oncological margin clearance, an intraoperative specimen radiograph was taken to confirm that the marker coil was localized satisfactorily within the specimen with adequate marginal clearance (Fig. 2). Magnetometer counts were repeated on

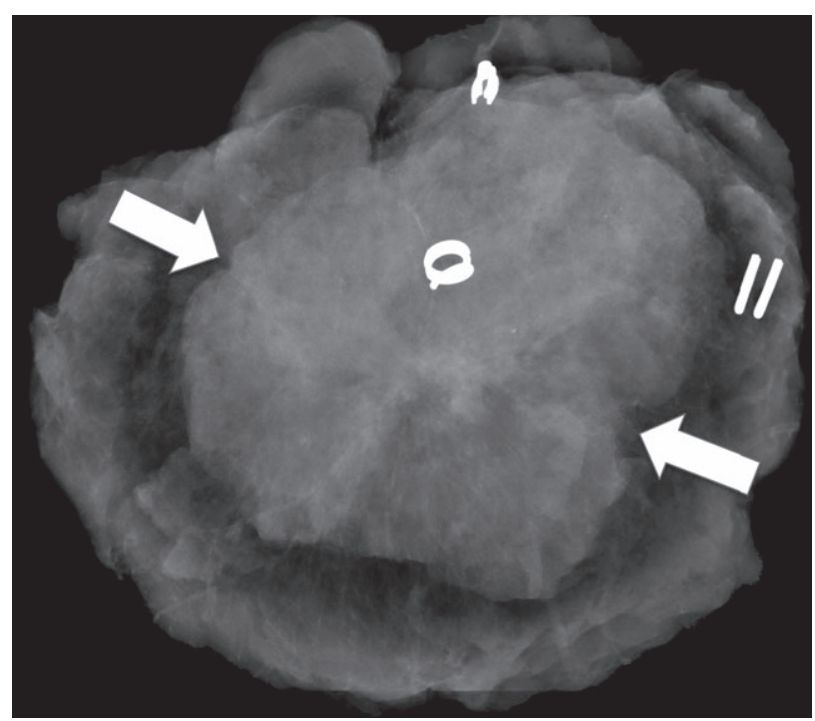

Fig. 2 Intraoperative specimen $\mathrm{X}$-ray demonstrating a cancer located in the centre of the excised specimen (arrows mark radiological tumour extent). A marker coil is visible near the tumour centre. Standard orientation clips are visible (2 at superior margin and 3 towards the nipple)

the excised specimen. If the surgeon was satisfied with the oncological clearance, the wound was closed; otherwise further cavity excisions were made. The specimen was marked in accordance with standard local protocols and all specimens were sent for histopathological assessment.

\section{Histopathology}

All histopathological evaluation was carried out according to local protocols. Inadequate excision of invasive cancer or incidental in situ disease was defined by margins of less $2 \mathrm{~mm}$ at the outset of the trial according to the standard local protocols at the lead site (Guy's Hospital). These local protocols were changed to less than $1 \mathrm{~mm}$ for invasive and less than $2 \mathrm{~mm}$ for in situ disease in March 2014, and this was the same as used at the second site (Cardiff). Nodes were reported as normal or containing macrometastases (larger than $2 \mathrm{~mm}$ ), micrometastases $(2 \mathrm{~mm}$ or less but larger than $0.2 \mathrm{~mm})$ and isolated tumour cells $(0.2 \mathrm{~mm}$ or smaller). The size of the largest metastatic deposit was recorded.

\section{Predefined endpoints}

The primary endpoint was defined as successful localization of breast cancer within excised specimens (peak magnetometer count within the excised specimen; corroboration with the skin marking overlying the lesion after excision in patients who underwent mastectomy). 
Secondary endpoints were excised specimen margin status; volume of excised specimens; calculated resection ratio (CRR), defined as total resection volume $(\mathrm{TRV}) /$ optimal resection volume $(\mathrm{ORV})^{15}$, and sentinel lymph node (SLN) identification with each technique.

\section{Results}

A total of 33 procedures were performed in 32 patients (1 patient had bilateral breast cancer). The first 13 procedures were done in symptomatic patients with palpable breast cancers, of whom three underwent mastectomy. The subsequent 20 patients had clinically occult, non-palpable breast cancers. Patient and tumour characteristics are shown in Table 1.

Table 1 Patient and tumour characteristics

\begin{tabular}{|c|c|}
\hline & No. of tumours* \\
\hline \multicolumn{2}{|l|}{ Mammographic screen-detected } \\
\hline Yes & 13 \\
\hline No & 20 \\
\hline \multicolumn{2}{|l|}{ Age (years) $\dagger$} \\
\hline $29-50$ & 3 \\
\hline $51-69$ & 24 \\
\hline$\geq 70$ & 5 \\
\hline \multicolumn{2}{|l|}{ Type of surgery } \\
\hline Mastectomy & 6 \\
\hline Breast-conserving surgery & 27 \\
\hline \multicolumn{2}{|l|}{ Node status by largest metastasis } \\
\hline No metastasis & 27 \\
\hline ITC & 1 \\
\hline Micrometastasis & 2 \\
\hline Macrometastasis & 3 \\
\hline \multicolumn{2}{|l|}{ Lymphovascular invasion } \\
\hline Yes & 2 \\
\hline No & 31 \\
\hline \multicolumn{2}{|l|}{ Tumour grade } \\
\hline 1 & 8 \\
\hline 2 & 21 \\
\hline 3 & 4 \\
\hline \multicolumn{2}{|l|}{ Tumour size } \\
\hline $\mathrm{T} 1$ & 23 \\
\hline T2 & 8 \\
\hline T3 & 2 \\
\hline \multicolumn{2}{|l|}{ Oestrogen receptor status } \\
\hline Positive & 28 \\
\hline Negative & 5 \\
\hline \multicolumn{2}{|l|}{ HER2 status } \\
\hline Positive & 2 \\
\hline Negative & 31 \\
\hline \multicolumn{2}{|l|}{ Tumour type } \\
\hline Invasive, no special type & 19 \\
\hline Invasive, pure special type & 11 \\
\hline Mixed-lobular and no special type & 1 \\
\hline Mixed-other and no special type & 2 \\
\hline
\end{tabular}

*Unless indicated otherwise; †number of patients. ITC, isolated tumour cells; HER2, human epidermal growth factor receptor 2.

\section{Lesion localization}

Radiological intratumoral injection of $0.5 \mathrm{ml}$ magnetic tracer was performed successfully in all 32 patients.

Table 2 Intraoperative localization and surgical excision of breast cancers

$\begin{array}{lc}\text { No. of patients } & 32 \\ \text { No. of cancers treated } & 33 \\ \text { Successful localization of tumour at surgical excision site } \dagger & 33 \\ \text { Non-palpable cancers } & 20 \\ \quad \text { Surgical margin status for invasive } \pm \text { in situ disease } & \\ \quad \text { Clear } & 18 \\ \quad \text { Close } & 0 \\ \quad \text { Involved } & 2 \\ \text { Optimal resection volume }\left(\mathrm{cm}^{3}\right)^{\star} & 22 \cdot 5(12 \cdot 4) \\ \text { Total resection volume }\left(\mathrm{cm}^{3}\right)^{*} & 49 \cdot 5(30 \cdot 6) \\ \text { Calculated resection ratio } & 2 \cdot 5(2 \cdot 2)\end{array}$

*Values are mean(s.d.). $†$ Retained peak magnetometer count at site of original injection under ultrasound guidance.

Table 3 Identification (detection) rates and discordant procedures by method of detection

\begin{tabular}{lccc} 
& \multicolumn{2}{c}{ Radioisotope + blue dye } & \\
\cline { 2 - 3 } & $\begin{array}{c}\text { Failed } \\
\text { detection }\end{array}$ & $\begin{array}{c}\text { Successful } \\
\text { detection }\end{array}$ & Total \\
\hline Magnetic technique & & & \\
$\quad$ Failed detection & 0 & 5 & 5 \\
$\quad$ Successful detection & 1 & 27 & 28 \\
$\quad$ Total & 1 & 32 & 33 \\
Magnetic technique + blue dye & & & \\
$\quad$ Failed detection & 0 & 1 & 1 \\
Successful detection & 1 & 31 & 32 \\
Total & 1 & 32 & 33 \\
\hline
\end{tabular}

Discordance is six of 33 procedures for magnetic technique alone versus standard dual technique, and two of 33 for magnetic technique + blue dye versus standard dual technique.

Table 4 Number of nodes removed in the study cohort (33 tumours) and mean per tumour, by mode of detection

\begin{tabular}{|llrr} 
& \multicolumn{2}{c}{ Radioisotope + blue dye } & \\
\cline { 2 - 3 } & $\begin{array}{c}\text { Failed } \\
\text { detection }\end{array}$ & $\begin{array}{c}\text { Successful } \\
\text { detection }\end{array}$ & Total \\
\hline Magnetic technique & & & \\
$\quad$ Failed detection & $0(0)$ & $15(0.45)$ & $15(0.45)$ \\
$\quad$ Successful detection & $5(0.15)$ & $47(1.43)$ & $52(1.58)$ \\
$\quad$ Total & $5(0.15)$ & $62(1.88)$ & $67(2.03)$ \\
Magnetic technique +blue dye & & & \\
$\quad$ Failed detection & $0(0)$ & $7(0.21)$ & $7(0.21)$ \\
$\quad$ Successful detection & $5(0.15)$ & $55(1.67)$ & $60(1.82)$ \\
$\quad$ Total & $5(0.15)$ & $62(1.88)$ & $67(2.03)$
\end{tabular}

Values are number of tumours removed in the study cohort, with mean per tumour in parentheses. Discordance is 20 (30 per cent) of 67 nodes for magnetic technique alone versus standard dual technique, and 12 (18 per cent) of 67 for magnetic technique + blue dye versus standard dual technique. 


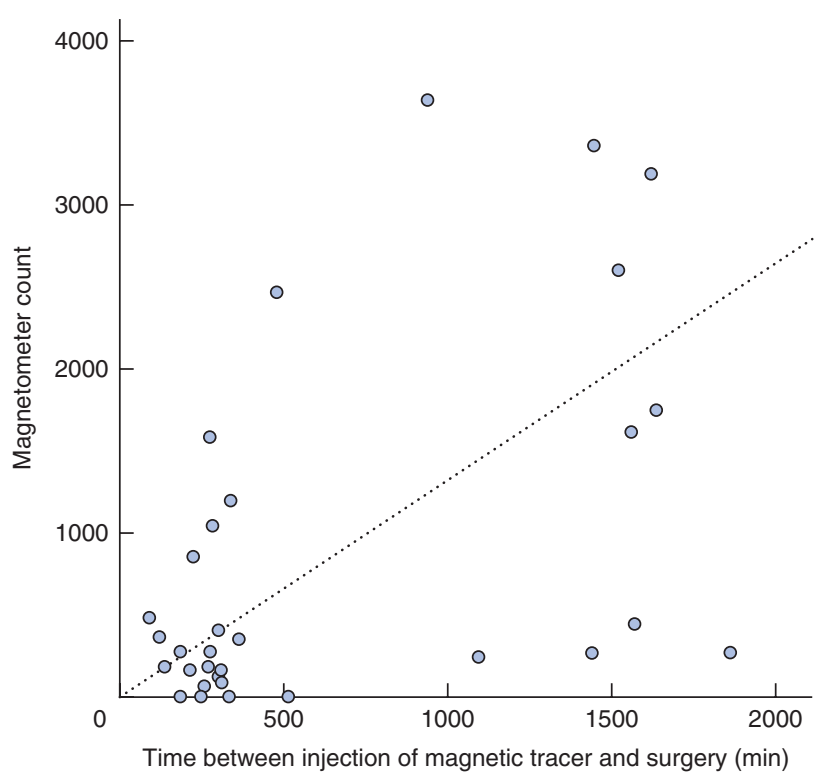

Fig. 3 Relationship between ex vivo magnetometer count of the 'hottest' node retrieved for each patient and time between injection of the magnetic tracer and surgery

Successful intraoperative localization, corresponding to the presence of peak magnetometer counts within the excised specimens, was also achieved in all instances (Table 2). Spillage of iron oxide at the skin puncture site on withdrawal of the needle posed a technical challenge. This was recognized and avoided by aspiration of the syringe before removal of the needle from the tumour centre. Two of the 20 patients with non-palpable breast cancers required a surgical re-excision (second operation) owing to the presence of incidental in situ disease at the excised specimen margins. The mean(s.d.) CRR was $2 \cdot 5(2 \cdot 2)$ for the non-palpable lesions (Table 2).

\section{Sentinel lymph node biopsy}

The SLN was identified successfully in 28 of 33 procedures ( 28 of 32 patients) by the magnetic technique alone, 32 of 33 procedures by the magnetic and blue dye techniques combined, and 32 of 33 procedures with the standard dual technique (Table 3). Discordance between the standard and magnetic techniques was observed in six of 33 procedures and 20 (30 per cent) of 67 nodes retrieved (Table 4). This was reduced to two of 33 procedures, and 12 (18 per cent) of 67 nodes respectively with the addition of blue dye to the magnetic technique. The single failed SLNB procedure using the standard technique and five using the magnetic technique proved to be negative on final histology. Of the 33 procedures performed, there were five instances of nodal involvement, two owing to micrometastases and three macrometastases. All macrometastases were identified independently by both techniques, but one micrometastasis was identified only using the magnetic technique. Of the 67 nodes excised, 52 'true' sentinel nodes were identified by the magnetic technique alone, 60 by the magnetic technique combined with blue dye, and 62 by the standard dual technique (Table 4). The overall retrieval rate was 2.03 nodes per procedure, 1.58 nodes for the magnetic technique alone, 1.82 nodes for the magnetic technique combined with blue dye, and 1.88 nodes for the standard dual technique.

Fig. 3 shows that the ex vivo sentinel node count was highest when tracer was injected the day before operation.

\section{Discussion}

The advent of screening programmes for breast cancer and the development of advanced imaging modalities such as MRI for diagnostic purposes has resulted in an increase in the identification of non-palpable breast cancers. Despite this increase in the burden of disease, current techniques that allow localized surgical excision suffer from technical difficulties, resulting in high re-excision rates ${ }^{3}$ as well as logistical drawbacks; these prevent uptake ${ }^{2,3}$. It is therefore imperative that new techniques are developed to overcome current limitations.

The first feasibility study for a novel application of a magnetic tracer for breast cancer localization and also evaluating concurrent SLNB is presented here. This technique does not require use of a wire and radioisotopes. All breast cancers were localized successfully in this series of 32 patients (33 procedures). It was predefined in the study protocol that at least ten of the patients initially recruited had to have palpable breast cancers that were visible on ultrasound examination. This was necessary to confirm the ability of the magnetic tracer to maintain a peak magnetometer count at the site of injection (within the centre of the breast cancer), as demonstrated previously in vivo ${ }^{13}$. All breast surgical localization procedures were carried out at least $2 \mathrm{~h}$ after injection of the magnetic tracer. One patient underwent SLNB before autologous breast reconstruction and the subsequent breast surgical localization was performed successfully 7 days after injection of the magnetic tracer. For all tumours the peak magnetometer counts were present at the original injection site, within the centre of the palpable cancer.

Once it had been confirmed that safe localization of tumour by magnetic tracer was feasible, progression was made to the localization of non-palpable breast cancers. The 20 patients with non-palpable breast cancers all underwent successful surgical excision guided by peak magnetometer counts. Surgical re-excision (second operation) 
was required in two patients owing to margin involvement with incidental in situ disease. These findings clearly demonstrate the ability of this magnetic technique safely to localize non-palpable lesions, allowing adequate excision. The ORV and TRV volumes were computed, and applied to create a CRR ${ }^{15}$. The mean(s.d.) CRR value was $2 \cdot 5(2 \cdot 2)$, which compared favourably with results for wire-guided localization, intraoperative ultrasonography and radioguided occult lesion localization, for which median values of $2 \cdot 8,3 \cdot 2$ and $3 \cdot 8$ respectively have been recorded ${ }^{15}$. This provides early support that the magnetic technique does not result in excision of an excessive tissue volume.

SLNB is the standard procedure for axillary staging in early breast cancer ${ }^{8,16-20}$. Intratumoral injection of the magnetic tracer allowed localization and concurrent SLNB to be performed successfully in 28 of 33 procedures. The SLN identification rate was higher when the standard dual technique with a periareolar subcutaneous injection was used, with successful identification in 32 of 33 procedures. There was discordance in SLN identification between the magnetic and standard dual technique in six of 33 procedures. The SLN identification rate in previous trials ${ }^{7,8}$ of the magnetic technique performed only for SLNB in breast cancer, with periareolar injections of magnetic tracer and radioisotope, with or without blue dye, varied between 94 and 98 per cent with discordance rates of $2-6.8$ per cent. Therefore, in the present study the location of the injection of the magnetic tracer is most likely responsible for the inferior SLN identification rate for the magnetic technique compared with the standard dual technique and the observed high discordance rates.

When the magnetic tracer was combined with blue dye, SLN identification was successful in 32 of 33 procedures overall. This is consistent with studies ${ }^{21-24}$ that applied a single intratumoral injection of radioisotope for sentinel node and occult lesion localization, which reported SLN identification rates between 79 and 91 per cent when radioisotope alone was used, rising to 90-98 per cent with the addition of periareolar injection of blue dye. Here, the overall and mean number of sentinel nodes excised was found to be similar with the magnetic technique (mean 1.58 nodes for magnetic technique alone; 1.82 nodes with addition of blue dye) compared with the dual technique (1.88 nodes). This is consistent with previous studies ${ }^{7-9}$ for magnetic SLNB, which recorded retrieval of about two nodes per procedure for the magnetic technique.

The magnetic tracer was shown to remain localized for 1 week, after which peak magnetometer counts were still present at the injection site at surgery, although this was tested only in a single patient. Should the retention of magnetic tracer at the site of injection be further confirmed beyond this time frame, the technique could potentially be applied as a marker after breast biopsy, or even before primary chemotherapy, and could subsequently be used for surgical excision without additional wire localization.

Concurrent SLNB performed after intratumoral injection of magnetic tracer demonstrated an inferior SLN identification rate compared with the standard dual technique. However, when blue dye was also included with the magnetic tracer the identification rates were similar (success in 32 of 33 procedures). This suggests that, with the present technique, intratumoral injection of magnetic tracer on its own without blue dye would not provide satisfactory SLN identification. The highest ex vivo sentinel node counts were recorded in patients who underwent injection of the magnetic tracer the day before surgery. This suggests that lymphatic uptake of magnetic tracer from the injection site to the sentinel node continues with time. Therefore, the SLN identification rate may be improved by administering the localized injection the day before surgery or even earlier. Alternatively, magnetic SLNB identification could be improved by administering an additional periareolar injection of magnetic tracer in the operating theatre, as in the SentiMAG Multicentre Trial ${ }^{7}$.

The MagSNOLL Trial has demonstrated that the magnetic technique is feasible for breast cancer localization and concurrent SLNB. This technique overcomes the limitations of wire-guided and radioguided surgery for lesion localization. Further studies should evaluate localization with lower volumes of tracer at least a day before surgery.

\section{Collaborators}

Other members of the MagSNOLL Trialists Group are: H. Hamed (Guy's and St Thomas' NHS Foundation Trust, London), A. Kothari (Guy's and St Thomas' NHS Foundation Trust, London), T. Kovacs (Guy's and St Thomas' NHS Foundation Trust, London), S. McWilliams (Guy's and St Thomas' NHS Foundation Trust, London), I. Monypenny (University Hospital Llandough, Cardiff), C. Morris (University Hospital Llandough, Cardiff), S. Pinder (King's College London, London), A. Purushotham (King's College London, London) and J. Scudder (Guy's and St Thomas' NHS Foundation Trust, London).

\section{Acknowledgements}

The study was sponsored by King's College London and Guy's and St Thomas' NHS Foundation Trust. The authors thank Endomagnetics for providing the magnetic tracer and equipment support, and the UK National 
Institute for Health Research for adopting and supporting the MagSNOLL Trial.

Although Q.A.P. fulfils a part-time paid advisory role as Chief Scientist (Physics) for the magnetic SLNB company Endomagnetics, his role in this work has been purely academic.

Disclosure: The authors declare no other conflict of interest.

\section{References}

1 Lovrics PJ, Cornacchi SD, Farrokhyar F, Garnett A, Chen $\mathrm{V}$, Franic $\mathrm{S}$ et al. The relationship between surgical factors and margin status after breast-conservation surgery for early stage breast cancer. Am 7 Surg 2009; 197: 740-746.

2 Ahmed M, Douek M. Intra-operative ultrasound versus wire-guided localization in the surgical management of non-palpable breast cancers: systematic review and meta-analysis. Breast Cancer Res Treat 2013; 140: 435-446.

3 Ahmed M, van Hemelrijck M, Douek M. Systematic review of radioguided versus wire-guided localization in the treatment of non-palpable breast cancers. Breast Cancer Res Treat 2013; 140: 241-252.

4 Ahmed M, Abdullah N, Cawthorn S, Usiskin SI, Douek M. Why should breast surgeons use ultrasound? Breast Cancer Res Treat 2014; 145: 1-4.

5 Rescigno J, Zampell JC, Axelrod D. Patterns of axillary surgical care for breast cancer in the era of sentinel lymph node biopsy. Ann Surg Oncol 2009; 16: 687-696.

6 Joshi TP, Pankhurst QA, Hattersley S, Brazdeikis A, HallCraggs M, De Vita E et al. Magnetic nanoparticles for detecting sentinel lymph nodes. Eur 7 Surg Oncol 2007; 33: 1135 .

7 Douek M, Klaase J, Monypenny I, Kothari A, Zechmeister $\mathrm{K}$, Brown D et al. Sentinel node biopsy using a magnetic tracer versus standard technique: the SentiMAG Multicentre Trial. Ann Surg Oncol 2014; 21: 1237-1245.

8 Thill M, Kurylcio A, Welter R, van Haasteren V, Grosse B, Berclaz G et al. The Central-European SentiMag study: Sentinel lymph node biopsy with superparamagnetic iron oxide (SPIO) vs. radioisotope. Breast 2014; 23: 175-179.

9 Shiozawa M, Lefor AT, Hozumi Y, Kurihara K, Sata N, Yasuda $\mathrm{Y}$ et al. Sentinel lymph node biopsy in patients with breast cancer using superparamagnetic iron oxide and a magnetometer. Breast Cancer 2013; 20: 223-229.

10 Ahmed M, Purushotham AD, Douek M. Novel techniques for sentinel lymph node biopsy in breast cancer: a systematic review. Lancet Oncol 2014; 15: e351-e362.

11 Johnson L, Pinder SE, Douek M. Deposition of superparamagnetic iron-oxide nanoparticles in axillary sentinel lymph nodes following subcutaneous injection. Histopathology 2013; 62: 481-486.

12 Ahmed M, de Rosales RT, Douek M. Preclinical studies of the role of iron oxide magnetic nanoparticles for nonpalpable lesion localization in breast cancer. 7 Surg Res 2013; 185: 27-35.
13 Anninga B, Ahmed M, Van Hemelrijck M, Pouw J, Westbroek D, Pinder S et al. Magnetic sentinel lymph node biopsy and localization properties of a magnetic tracer in an in vivo porcine model. Breast Cancer Res Treat 2013; 141: $33-42$.

14 UK Clinical Research Network. Magnetic Sentinel Node and Occult Lesion Localisation (MagSNOLL). http://public. ukcrn.org.uk/search/StudyDetail.aspx?StudyID=14979 [accessed 17 October 2014].

15 Krekel NM, Zonderhuis BM, Stockmann HB, Schreurs WH, van der Veen H, de Lange de Klerk ES et al. A comparison of three methods for nonpalpable breast cancer excision. Eur F Surg Oncol 2011; 37: 109-115.

16 Gill G; SNAC Trial Group of the Royal Australasian College of Surgeons (RACS) and NHMRC Clinical Trials Centre. Sentinel-lymph-node-based management or routine axillary clearance? One-year outcomes of sentinel node biopsy versus axillary clearance (SNAC): a randomized controlled surgical trial. Ann Surg Oncol 2009; 16: 266-275.

17 Kim T, Giuliano AE, Lyman GH. Lymphatic mapping and sentinel lymph node biopsy in early-stage breast carcinoma: a metaanalysis. Cancer 2006; 106: 4-16.

18 Krag DN, Anderson SJ, Julian TB, Brown AM, Harlow SP, Ashikaga $\mathrm{T}$ et al. Technical outcomes of sentinel-lymph-node resection and conventional axillary-lymph-node dissection in patients with clinically node-negative breast cancer: results from the NSABP B-32 randomised phase III trial. Lancet Oncol 2007; 8: 881-888.

19 Veronesi U, Paganelli G, Viale G, Luini A, Zurrida S, Galimberti $\mathrm{V}$ et al. A randomized comparison of sentinel-node biopsy with routine axillary dissection in breast cancer. N Engl 7 Med 2003; 349: 546-553.

20 Zavagno G, De Salvo GL, Scalco G, Bozza F, Barutta L, Del Bianco $\mathrm{P}$ et al. A randomized clinical trial on sentinel lymph node biopsy versus axillary lymph node dissection in breast cancer: results of the Sentinella/GIVOM trial. Ann Surg 2008; 247: 207-213.

21 Chow MP, Hung WK, Chu T, Lui CY, Ying M, Mak KL et al. Isotope-guided surgery for nonpalpable breast cancer. World f Surg 2011; 35: 165-169.

22 Giacalone PL, Bourdon A, Trinh PD, Taourel P, Rathat G, Sainmont M et al. Radioguided occult lesion localization plus sentinel node biopsy (SNOLL) versus wire-guided localization plus sentinel node detection: a case control study of 129 unifocal pure invasive non-palpable breast cancers. Eur F Surg Oncol 2012; 38: 222-229.

23 van Rijk MC, Tanis PJ, Nieweg OE, Loo CE, Olmos RA, Oldenburg HS et al. Sentinel node biopsy and concomitant probe-guided tumor excision of nonpalpable breast cancer. Ann Surg Oncol 2007; 14: 627-632.

24 Mariscal Martínez A, Solà M, de Tudela AP, Julián JF, Fraile M, Vizcaya S et al. Radioguided localization of nonpalpable breast cancer lesions: randomized comparison with wire localization in patients undergoing conservative surgery and sentinel node biopsy. A7R Am 7 Roentgenol 2009; 193: 1001-1009. 